\title{
Gene of the month: DICER1: ruler and controller
}

\author{
Michelle Thunders (1), Brett Delahunt
}

Pathology and Molecular Medicine, University of Otago, Wellington, New Zealand

\section{Correspondence to}

Dr Michelle Thunders, Pathology and Molecular Medicine,

University of Otago, Wellington 6242, New Zealand; michelle. thunders@otago.ac.nz

Accepted 28 October 2020 Published Online First 8 December 2020

\begin{abstract}
DICER1 is a highly conserved RNaselll endoribonuclease that has a critical role in the biogenesis of microRNAs (miRNAs). miRNAs are small regulatory RNAs responsible for post-transcriptional gene silencing, controlling more than half of human protein-coding genes. This is achieved through the targeting and regulation of complementary RNA transcripts and has a welldocumented role in post-transcriptional gene regulation and transposon repression. DICER1 deficiency results in dysregulation of miRNAs, changing the expression of many genes. DICER1 syndrome represents a collection of benign and malignant tumours arising from an autosomally inherited germline mutation leading to an inherited predisposition to cancer. The syndrome represents an unusual form of Knudson's two-hit hypothesis, where individuals with a pathogenic germline DICER 1 variant acquire a second trans-somatic missense DICER 1 mutation. This somatic mutation appears to have to occur in one of five hotspots codons and may contribute towards the incomplete penetrance observed within DICER1 syndrome families. In this case, DICER 1 is haploinsuffcient with only one deletion required and partial loss of function being advantageous to tumours over complete loss of function. As increasing data emerge reaffirming the pivotal role of DICER1 in the maintenance of human physiology, DICER 1 is likely to become an increasingly attractive target for novel therapeutic strategies.
\end{abstract}

\section{DICER1 GENE FUNCTION}

DICER1 is essential for the maturation of almost all microRNAs (miRNAs) . ${ }^{1}$ It is a highly conserved double-stranded RNaseIII endoribonuclease and has a critical role in post-transcriptional gene silencing. DICER1 cleaves precursor miRNAs (pre-miRNA) to produce 20-22 nucleotide-long mature regulatory miRNAs and short interfering RNAs (SiRNAs). SiRNAs and miRNAs act as guide molecules in RNA interference (RNAi) with the RNA-induced silencing complex (RISC) targeting sequenced matched targets in the three prime untranslated region (3'UTR) of specific mRNAs, to degrade them or prevent their translation. ${ }^{1} \mathrm{RNAi}$ is a method of gene regulation employed widely across eukaryotes whereby dsRNA can silence genes. DICER1 has at least three distinct roles in RNAi pathways; processing pre-miRNAs to miRNAs and long dsRNA into siRNAs, loading small RNAs onto Argonaute proteins (AGO) to programme the RISC (RISC loading) and scaffolding protein-protein interactions between RNAi cofactors in the RISCloading complex, the RISC and other complexes involved in endogenous RNAi processes. ${ }^{2}$ RNA interference is a particularly powerful method of gene control as each miRNA can target a large number of transcripts due to suboptimal complementarity. This is achieved through target and regulation of complementary RNA transcripts and has a well-documented role in post-transcriptional gene regulation and transposon repression. While this is important in the control and elimination of transcripts from mobile and repetitive DNA elements of the genome, it is also important in the degradation of exogenous RNA, for example, of viral origin. ${ }^{3}$

RNA interference is particularly important in neurogenesis, during embryonic development, when the utmost precision is required in terms of spatio-temporal control of gene expression. Experiments in mice show loss of DICER1 results in severe disruption to neuronal cell differentiation. ${ }^{2}$ Both Argonaut and DICER1 have an evolutionary conserved role in chromosome function, including guiding chromatin modification and promoting chromosome segregation in mitosis and in meiosis regulating chromosome and genomic dosage. They also have a highly important role in genome defence against viruses, transposons development and cellular differentiation, tumour progression and in the resolution of DNA damage. It is believed that the ancestral role of DICER1 is more associated with genome defence than with gene regulation. ${ }^{4}$ Viruses have an inbuilt counter measure to RNA interference and often encode viral suppressors of RNAi. A recent report has suggested that SARS-CoV-2 suppresses RNAi through preventing the recognition and cleavage of viral dsRNA by DICER 1,5 a key way of evading the human immune system that could contribute to the pathogenicity of this particularly problematic coronavirus. DICER1 is most known for its role in DICER1 syndrome. The DICER1 syndrome is a constellation of benign and malignant tumours whose clinical expression commences in childhood. ${ }^{6}$ In cancer, deregulation of miRNAs has a pro-oncogenic effect, the overexpression of one miRNA may act to inhibit the protein translation of a tumour suppressor gene, while the down regulation of another miRNA may increase the protein level of an oncogene. ${ }^{7}$

\section{GENE STRUCTURE}

Located on chromosome 14q32.13 the DICER1 gene has 27 exons and encodes a 1922 amino acid protein approximately $200 \mathrm{kDa}$. DICER 1 in the mouse is found on the mouse chromosome $12 \mathrm{E}$. DICER1 is composed of three structurally rigid regions; RNase, Platform and PAZ connected by flexible hinges. The RNase region is made up of the two functional enzymatic domains RNAase III domains (RNaseIIIa and RNaseIIIb) and the bridging domain. The platform region is composed of the platform domain and the connector helix, 


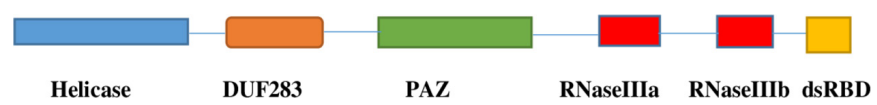

Figure 1 Schematic of the protein domains in DICER1.

and the third region is the PAZ domain which binds the end of dsDNA. The distance between the PAZ and the RNaseIIIa domain equates the length of 25 base pairs of RNA making DICER1 a molecular ruler recognising dsRNA and cleaving at a fixed specific distance from the helical end. As illustrated in figure 1, the DICER1 protein domains are ordered as follows from the $\mathrm{N}$ to the $\mathrm{C}$ terminal: Helicase domain (comprising DEXD/H, TRBP-BD and HELICc), DUF283 domain, PAZ domain (comprising Piwi/Argonaute/Zwille), RNaseIIIa, RNaseIIIb and dsRBD. ${ }^{3}$ The DNase activity of the C-terminal fragment is critical for DNA fragmentation during apoptosis. ${ }^{1}$

\section{GENE MUTATIONS}

DICER1 syndrome germline mutations can occur anywhere in the DICER1 gene but typically result in loss of function of that gene copy and the prevalence of loss of function germline DICER1 mutations has been estimated to be $1 / 10600$ in the general population. ${ }^{6}$ Most carriers with a germline DICER1 variant live healthy lives, although with an increased risk of malignancy. ${ }^{7}$ Mutations in the DICER1 gene are associated with aberrant cell proliferation and deregulation of cell growth by genes that typically regulate gonadogenesis. Approximately $87 \%$ of germline mutations are inherited in an autosomal dominant manner with incomplete penetrance, while $13 \%$ arise as de novo mutations, both resulting in improper cleavage and loss of DICER1 function. ${ }^{8}$

In the DICER1 syndrome, the related tumours typically acquire a second somatic mutation. The DICER1 syndrome, therefore, follows Knudson's two-hit hypothesis but, there are exceptions, as the second mutation does not fully take out DICER1 function, more so, it affects DICER1's ability to process miRNAs, altering its function in specific cell types. The dysfunctional DICER1 protein typically producing an abnormal mix of miRNAs. These somatic missense variations are typically found in five 'hotspot' codons in the RNaseIIIb domain (E1705, D1709, G1809, D1810, E1813) altering the cleavage capability of the protein and have been identified in almost all DICER1associated tumours. Dysfunction of gene regulation, based on RNA interference, is a common feature in the pathogenesis of cancer so given its crucial role in miRNA and siRNA biogenesis, DICER1 is an important gene to study from the carcinogenesis perspective. In cancer, widespread downregulation of miRNAs is a common observation. Supressing DICER1 enhances tumourigenic activities, supporting DICER1 as a haploinsufficient tumour suppressor. ${ }^{9}$ The knocking down of DICER1 expression suppress growth and tumourigenic capacity of human prostate cell lines, but enhances migratory capacities. ${ }^{10}$

\section{GENE EXPRESSION}

Copy number alterations of miRNAs and their regulatory genes are highly prevalent in cancer, particularly high copy abnormalities of DICER1 and AGO2. ${ }^{7}$ Additionally, Dobrijević et al identified the SNP rs1057035 as a potential biomarker associated with the risk of developing cancer independent of DICER1 syndrome, with the minor $\mathrm{C}$ allele protective against cancer susceptibility. ${ }^{11}$ DICER1 deficiency results in the dysregulation of miRNAs which, in turn, changes the expression of many genes. In mouse the loss of DICER1 function blocks miRNA biogenesis and impedes embryonic development. In experiments looking at conditional DICER1 knockdowns, effects include reduced proliferation of neural progenitor cells, delayed cell cycle kinetics, dysregulated neuronal migration and differentiation, activated caspase 3 to induce apoptosis and induction of astrocyte differentiation, resulting in embryonic lethality, abnormal neuronal development and disrupted nervous system function. ${ }^{12}$ DICER1 deletions in the developing nervous system produce different contradictory outcomes dependent on the timing of the deletion. Most commonly, the response to DICER1 ablation is increased apoptosis and senescence. Other consequences of DICER 1 ablation is that it severely affects limb morphogenesis, causes skin tissue disorganisation, impairment of embryonic stem cell differentiation and attenuation of specified functionality of differentiated B lymphocytes.

Cellular stress response also has a role in the control of DICER1 complex activity via interactions with stress granules. Under stress, DICER1 and AGO2 interact more with RNA binding proteins that are normally found in stress granules. Experiments, using chemical stress inducers to bring about the induction of stress granules, resulted in reduced DICER1 activity, overexpression of stress granule resident proteins and mutant amyotrophic lateral sclerosis-causing proteins. The opposite was observed when stress granule formation was blocked and miRNA biogenesis recovered. ${ }^{13}$ Erroneous expression of DICER1 has also been associated with autoimmune disorders with both loss and upregulation contributing to a range of pathologies including ankylosing spondylitis, psoriasis, rheumatoid arthritis, multiple sclerosis and autoimmune thyroid disorders. ${ }^{14}$ Potentially more subtle effects of timing and extent of gene expression during embryogenesis could contribute to human facial variation as, in a study by Liu et al, SNP rs7161418 in DICER1 was one of 12 SNPS from 10 genes significantly associated with facial morphology in the Han Chinese populations. ${ }^{15}$

Ageing is associated with decreased expression of DICER1 and changes in its diurnal expression pattern. ${ }^{16}$ Impairment of DICER1 can result in accumulation of Alu RNA. Alu transposable elements (typically $300 \mathrm{bp}$ length) are the most common mobile element in the human genome, making up a $10 \%$ contribution to the genome. Alu transcripts are kept at a low level, up to 1000 per cell so there must be a mechanism controlling this, if not there would be pathogenic consequences. It is not clear what this mechanism is, but may include endonucleolytic processing of $A l u$ transcripts by DICER1. A recent study has reported that DICER1-dependent degradation of Alu RNAs is crucial for ameliorating detrimental effects in both human and mouse retinal cells. ${ }^{16}$ Accumulation of Alumers is associated with loss of mitochondrial integrity, release of mitochondrial nucleic acids and the activation of the inflammasome and cell death. ${ }^{17}$ DICER1 forms a heterodimer with a double-stranded RNA editing enzyme, Adenosine Deaminase RNA Specific (ADAR) and is targeted towards destroying Alumers. The heterodimer regulates the switch from RNA to protein-centric immunity

\section{DICER1 SYNDROME}

By the age of 10 years, 5.3\% of DICER1 germline mutation carriers develop a DICER1-associated neoplasm. By 20 years of age this has risen to $9.4 \%$ and by 50 years of age the incidence is $31.5 \% .{ }^{18}$ A significant percentage of individuals with the DICER1 syndrome have macrocephaly, additionally structural renal and ocular abnormalities are found as non-tumour expression. ${ }^{19}$ The most common tumours associated with the DICER1 syndrome are cystic nephroma and pleuropulmonary 


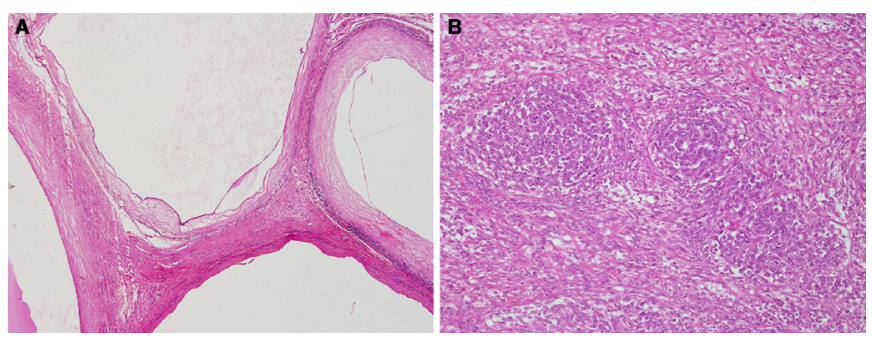

Figure 2 Histology from the DICER1 index family. ${ }^{21}$ (A) Cystic nephroma consisting of noncommunicating epithelial lined cysts with dense fibrous septa. (B) Pleuropulmonary blastoma comprising nests of blastema-like cells within sheets ofprimitive mesenchymal cells.

blastoma (PPB). ${ }^{20}$ The initial report of a familial association of these tumours was in 1993 and this involved three siblings, two with cystic nephroma and the third with PPB (figure $2 \mathrm{~A}, \mathrm{~B}$ ). ${ }^{21}$ $\mathrm{PPB}$ is the most common paediatric lung neoplasm ${ }^{22}$ The tumour develops as multilocular cysts on the surface of the pleura, having aggregates of rhabdomyoblasts and primitive cartilage in the septa (PPB type 1). A proportion of PPB type 1 will develop a mixed solid and cystic morphology, with tumour nodules consisting of primitive cells and rhabdmyoblasts (PPB type 2). Eventually the sarcomatous component will completely overgrow the cystic structures to produce a solid tumour consisting of poorly differentiated sarcoma (PPB type 3 ). It is unknown if all PPB type 1 will eventually evolve into PPB type 2 and PPB type $3 .^{23}$

Cystic nephroma are benign multiloculated tumours of the kidney that occur most frequently in children, with $9.2 \%$ having a familial association with PBB. ${ }^{24}$ Histologically identical tumours are also seen in individuals $>30$ years of age with a strong female predominance. ${ }^{25}$ The cystic nephromas in this older group are sporadic and unrelated to the DICER1 syndrome. In a manner somewhat similar to $\mathrm{PBB}$, cystic nephroma may rarely undergo malignant progression and develop into a sarcoma consisting of poorly differentiated primitive cells, which is termed anaplastic sarcoma of kidney. ${ }^{26}$ Wilms tumour has been reported in association with PPB and in a recent case report DICER1 mutation was confirmed. ${ }^{25} 27$

DICER1 mutations have been reported in 50\%-60\% of Sertoli-Leydig cell tumours. ${ }^{28} 29$ These tumours are the most frequently encountered ovarian sex-cord stromal tumour in association with $\mathrm{PPB}$, while tumours resembling juvenile granulosa cell tumour have also been reported. ${ }^{30}$ Interestingly two siblings, who both carried germline DICER1 mutations (E1705K and C801X) developed ovarian SLCT and multinodular goitre simultaneously. ${ }^{31}$ Embryonal rhabdomysarcoma of the urogenital tract has been occasionally reported in patients with DICER1 mutations. Mutation of DICER1 was found in two cases of cervical embryonal rhabdomyosarcoma from a series of 52 tumours, ${ }^{32}$ while cervical embryonal rhabdomyosarcoma was seen in three families with DICER1 germline mutations. ${ }^{33}$ Ovarian embryonal rhabdomyosarcoma was associated with cystic nephroma and thyroid goitre in a 6-year-old girl with DICER1 mutations. ${ }^{34}$ Embryonal rhabdomyosarcoma of unspecified site and thyroid goitre was reported in the cousin of a 2-year-old woman previously diagnosed with PPB and cystic nephroma. ${ }^{35}$ Embryonal rhabdomyosarcoma of bladder was diagnosed in a 12-year-old girl at 6 months of age along with pulmonary cysts. ${ }^{36}$ She underwent enucleation of the right eye for a a ciliary body medulloepithelioma when aged 3 years.
Ciliary body medulloepithelioma is a childhood embryonal tumour which may be teratoid or non-teratoid. Four cases of ciliary body medulloepithelioma were identified in in a review of a series of $\sim 600$ cases of PBB. Three tumours ( 1 teratoid and 1 non-teratoid), were in patients with $\mathrm{PBB}$. The ages of these patients ranging from 6 to 9 years, while the fourth tumours was in a patient's father. ${ }^{37}$ Ciliary body medulloepithelioma was diagnosed in a female at age 3.3 years who earlier been diagnosed with embryonal rhabdomyosarcoma of the bladder and lung cysts. ${ }^{36}$ A further case of ciliary body medulloepithelioma and $\mathrm{PBB}$ was reported from a child with germline DICER1 mutation. $^{28}$

Multinodular goitre is frequently see in individuals with DICER1 syndrome being diagnosed in 30\% of females and $13 \%$ of males by the age of 20 years. ${ }^{38}$ DICER 1 mutation also predisposes to a variety of thyroid tumours with follicular adenomas, follicular carcinomas, papillary carcinomas being reported. Somatic DICER1 mutations have also been associated with thyroid carcinosarcomas and teratomas. ${ }^{39}$ Recently somatic mutations of the DICER1 gene were seen in five of six patients diagnosed with poorly differentiated thyroid carcinoma. ${ }^{40}$ In DICER1 carrier females, there was found to be a high rate of developing DICER1-related multinodular goitre. ${ }^{41}$

Three children with PBB and cystic nephroma also had juvenile polyps of the small intestine. In two of the cases the polyps presented with intessusception. ${ }^{24}{ }^{42}$ DICER 1 mutation has also been reported in four patients with pineoblastoma from a series of 21 tumours. ${ }^{43}$ In this series, three of the patients were shown to have germline mutations. In a series of 13 cases of pituitary blastoma, there was a familial association with the DICER1 syndrome in five cases, while nine patients were shown to have DICER1 germline mutations. ${ }^{44}$ Other rare tumours associated with DICER1 mutations are peritoneal sarcoma, ${ }^{45}$ intracranial sarcoma $^{46}$ and nasal chondromesenchymal hamartoma. ${ }^{47}$

\section{CONCLUSION}

Although mostly known for its role in the DICER1 syndrome, DICER1 has a far wider and crucial role in control of gene expression. Loss or aberrant DICER1 expression contributes to a wide range of human pathologies including those that are autoimmune, neurological, reproductive and cardiovascular, with the pathogenesis largely due to deregulation of miRNA expression. Additionally, it is emerging that DICER1 has other functions unrelated to small RNA processing, such as roles in autophagosome formation, passive RNA binding, control of nuclear pore components and antiviral control. ${ }^{3}$ This makes DICER 1 an attractive target for development of novel treatments, not only for cancer but also for other human conditions whereby a multisystem approach could be a successful treatment strategy.

\section{Take home messages}

- DICER1 has an important role in the biogenesis of microRNAs

- DICER1 deficiency results in dysregulation of miRNAs, changing the expression of many genes.

- DICER1 syndrome represents a collection of benign and malignant tumours arising from a germline mutation leading to an inherited predisposition to cancer.

- The pivotal role of DICER1 in the maintenance of human physiology makes DICER1 an increasingly attractive target for novel therapeutic strategies. 
Handling editor Runjan Chetty.

Contributors MT and BD facilitated the conception and design of the project, drafted the manuscript and revised it critically for intellectual content.

Funding The authors have not declared a specific grant for this research from any funding agency in the public, commercial or not-for-profit sectors.

Competing interests None declared.

Patient consent for publication Not required.

Provenance and peer review Not commissioned; internally peer reviewed.

ORCID iDs

Michelle Thunders http://orcid.org/0000-0001-6722-7072

Brett Delahunt http://orcid.org/0000-0002-5398-0300

\section{REFERENCES}

1 Zhang J, Zhang X-H, Wang C-X, et al. Dysregulation of microRNA biosynthesis enzyme Dicer plays an important role in gastric cancer progression. Int I Clin Exp Pathol 2014;7:1702-7.

$2 \mathrm{Xu}$ Y, Hajdukiewicz K, Tiwari A, et al. Micrornas are indispensable for the proliferation and differentiation of adult neural progenitor cells in mice. Biochem Biophys Res Commun 2020;530:209-14.

3 Song M-S, Rossi JJ. Molecular mechanisms of Dicer: endonuclease and enzymatic activity. Biochem J 2017;474:1603-18.

4 Gutbrod MJ, Martienssen RA. Conserved chromosomal functions of RNA interference. Nat Rev Genet 2020:21:311-31.

$5 \mathrm{Mu}$ J, Xu J, Zhang L, et al. SARS-CoV-2-encoded nucleocapsid protein acts as a viral suppressor of RNA interference in cells. Sci China Life Sci 2020;63:1-4.

6 Guillerman RP, Foulkes WD, Priest JR. Imaging of DICER1 syndrome. Pediatr Radiol 2019;49:1488-505.

7 Zhang L, Huang J, Yang N, et al. microRNAs exhibit high frequency genomic alterations in human cancer. Proc Natl Acad Sci U S A 2006:103:9136-41.

8 Stewart DR, Best AF, Williams GM, et al. Neoplasm risk among individuals with a pathogenic germline variant in DICER1. J Clin Oncol 2019;37:668-76.

9 Kumar MS, Pester RE, Chen CY, et al. Dicer 1 functions as a haploinsufficient tumor suppressor. Genes Dev 2009;23:2700-4.

10 Zhang B, Chen H, Zhang L, et al. A dosage-dependent pleiotropic role of Dicer in prostate cancer growth and metastasis. Oncogene 2014;33:3099-108.

11 Dobrijević Z, Matijašević S, Išić Denčić T, et al. Association between genetic variants in DICER1 and cancer risk: an updated meta-analysis. Gene 2021;766:145132.

12 Nampoothiri SS, Rajanikant GK. Decoding the ubiquitous role of microRNAs in neurogenesis. Mol Neurobiol 2017;54:2003-11.

13 Eitan C, Hornstein E. Vulnerability of microRNA biogenesis in FTD-ALS. Brain Res 2016;1647:105-11.

14 Theotoki El, Pantazopoulou VI, Georgiou S, et al. Dicing the disease with Dicer: the implications of Dicer ribonuclease in human pathologies. Int J Mol Sci 2020;21:E7223.

15 Liu M, Li Y, Yang YF, et al. [Human facial shape related SNP analysis in Han Chinese populations]. Yi Chuan 2020;42:680-90.

16 Kaarniranta K. Pawlowska E, Szczepanska J, et al. DICER1 in the Pathogenesis of Age-related Macular Degeneration (AMD) - Alu RNA Accumulation versus miRNA Dysregulation. Aging Dis 2020;11:851-62.

17 Herbert A. Z-Dna and Z-RNA in human disease. Commun Biol 2019;2:7.

18 Stewart DR, Best AF, Williams GM, et al. Neoplasm Risk Among Individuals With a Pathogenic Germline Variant in DICER1. JCO 2019;37:668-76.

19 de Kock L, Wu MK, Foulkes WD. Ten years of DICER1 mutations: Provenance, distribution, and associated phenotypes. Hum Mutat 2019;40:1939-53.

$20 \mathrm{Kim}$ J, Schultz KAP, Hill DA, et al. The prevalence of germline DICER1 pathogenic variation in cancer populations. Mol Genet Genomic Med 2019;7:e555.

21 Delahunt B, Thomson KJ, Ferguson AF, et al. Familial cystic nephroma and pleuropulmonary blastoma. Cancer 1993:71:1338-42.

22 Messinger YH, Stewart DR, Priest JR, et al. Pleuropulmonary blastoma: a report on 350 central pathology-confirmed pleuropulmonary blastoma cases by the International pleuropulmonary blastoma registry. Cancer 2015;121:276-85.
23 Dehner LP, Schultz KA, Hill DA. Pleuropulmonary blastoma: more than a lung neoplasm of childhood. Mo Med 2019;116:206-10.

24 Boman F, Hill DA, Williams GM, et al. Familial association of pleuropulmonary blastoma with cystic nephroma and other renal tumors: a report from the International pleuropulmonary blastoma registry. J Pediatr 2006;149:850-4.

25 Caliò A, Eble JN, Grignon DJ, et al. Cystic nephroma in adults: a clinicopathologic study of 46 cases. Am J Surg Pathol 2016;40:1591-600.

26 Wu MK, Goudie C, Druker H, et al. Evolution of renal cysts to anaplastic sarcoma of kidney in a child with DICER1 syndrome. Pediatr Blood Cancer 2016;63:1272-5.

27 Abbo 0, Pinnagoda K, Brouchet L, et al. Wilms tumor, pleuropulmonary blastoma, and DICER1: case report and literature review. World I Surg Oncol 2018;16:164-8.

28 Slade I, Bacchelli C, Davies H, et al. Dicer1 syndrome: Clarifying the diagnosis, clinical features and management implications of a pleiotropic tumour predisposition syndrome. J Med Genet 2011;48:273-8.

29 Heravi-Moussavi A, Anglesio MS, Cheng S-WG, et al. Recurrent somatic DICER1 mutations in nonepithelial ovarian cancers. N Engl J Med 2012;366:234-42.

30 Oost EE, Charles A, Choong CS, et al. Ovarian sex cord-stromal tumors in patients with probable or confirmed germline DICER1 mutations. Int I Gynecol Pathol 2015:34:266-74.

31 Zhang Y, Ren M, Hong Y, et al. Sertoli-Leydig cell tumor in two siblings with DICER1 syndrome: a case report and literature review. Medicine 2020;99:e20806.

32 Doros L, Yang J, Dehner L, et al. DICER1 mutations in embryonal rhabdomyosarcomas from children with and without familial PPB-tumor predisposition syndrome. Pediatr Blood Cancer 2012;59:558-60.

33 Foulkes WD, Bahubeshi A, Hamel N, et al. Extending the phenotypes associated with DICER1 mutations. Hum Mutat 2011;32:1381-4.

34 de Kock L, Druker H, Weber E, et al. Ovarian embryonal rhabdomyosarcoma is a rare manifestation of the DICER1 syndrome. Hum Pathol 2015;46:917-22.

35 Fernández-Martínez L, Villegas JA, Santamaría Iñigo, et al. Identification of somatic and germ-line DICER1 mutations in pleuropulmonary blastoma, cystic nephroma and rhabdomyosarcoma tumors within a DICER1 syndrome pedigree. BMC Cancer 2017:17:146-151.

36 Fremerey J, Balzer S, Brozou T, et al. Embryonal rhabdomyosarcoma in a patient with a heterozygous frameshift variant in the DICER1 gene and additional manifestations of the DICER1 syndrome. Fam Cancer 2017;16:401-5.

37 Priest JR, Williams GM, Manera R, et al. Ciliary body medulloepithelioma: four cases associated with pleuropulmonary blastoma--a report from the International Pleuropulmonary Blastoma Registry. Br J Ophtha/mol 2011;95:1001-5.

38 Oliver-Petit I, Bertozzi A-I, Grunenwald S, et al. Multinodular goitre is a gateway for molecular testing of DICER1 syndrome. Clin Endocrinol 2019;91:669-75.

39 Nosé V. Dicer 1 gene alterations in thyroid diseases. Cancer Cytopathol 2020;128:688-9.

40 Chernock RD, Rivera B, Borrelli N, et al. Poorly differentiated thyroid carcinoma of childhood and adolescence: a distinct entity characterized by DICER1 mutations. Mod Pathol 2020;33:1264-74.

41 Merideth MA, Harney LA, Vyas N, et al. Gynecologic and reproductive health in patients with pathogenic germline variants in DICER1. Gynecol Oncol 2020;156:647-53.

42 Shaheen IS, Fitzpatrick M, Brownlee K, et al. Bilateral progressive cystic nephroma in a 9-month-old male infant requiring renal replacement therapy. Pediatr Nephrol 2010:25:1755-8.

43 de Kock L, Sabbaghian N, Druker H, et al. Germ-Line and somatic DICER1 mutations in pineoblastoma. Acta Neuropathol 2014;128:583-95.

44 de Kock L, Sabbaghian N, Plourde F, et al. Pituitary blastoma: a pathognomonic feature of germ-line DICER1 mutations. Acta Neuropathol 2014;128:111-22.

45 Schultz KAP, Nelson A, Harris AK, et al. Pleuropulmonary blastoma-like peritoneal sarcoma: a newly described malignancy associated with biallelic DICER1 pathogenic variation. Mod Pathol 2020;33:1922-9.

46 Kamihara J, Paulson V, Breen MA, et al. DICER1-associated central nervous system sarcoma in children: comprehensive clinicopathologic and genetic analysis of a newly described rare tumor. Mod Pathol 2020:33:1910-21.

47 Stewart DR, Messinger Y, Williams GM, et al. Nasal chondromesenchymal hamartomas arise secondary to germline and somatic mutations of DICER1 in the pleuropulmonary blastoma tumor predisposition disorder. Hum Genet 2014;133:1443-50. 